\title{
Fuzzy Linguistic Modelling Cognitive / Learning Styles for Adaptation through Multi-level Granulation
}

\author{
Ilham N. Huseyinov \\ Computer Engineering Department, European University of Lefke, \\ Gemikonagi-Lefke, Mersin 10, Turkey \\ ihuseynov@yahoo.com
}

\begin{abstract}
In this study, based on fuzzy linguistic modelling and fuzzy multi level granulation an adaptation strategy to cognitive/learning styles is presented. Fuzzy if-then rules are utilized to adaptively map cognitive/learning styles of users to their information navigation and presentation preferences through natural language expressions. The important implications of this approach are that, first, uncertain and vague information is handled; second, a mechanism for approximate adaptation at a variety of granulation levels is provided; third, a qualitative linguistic model of adaptation is presented. The proposed approach is close to human reasoning and thereby lowers the cost of solution, and facilitates the design of human computer interaction systems with high level intelligence capability.
\end{abstract}

Keywords: adaptive hypermedia, fuzzy granulation, cognitive/learning styles, navigation/presentation preferences, linguistic variables.

\section{Introduction}

The growing amount of information on the WEB and the heterogeneous characteristics of Web users have lead to a considerable attention to web-based adaptive hypermedia systems (WAHS) by the research community. The power of hypermedia of web technology is in its capability to support non-linear navigation in hyperspace and multimedia presentation of the web content. WAHS offers an alternative to the traditional "one-size-fits-all" hypermedia and Web systems by adapting to the goals, interests, and knowledge of individual users represented in the individual user models [1]. WAHS aims to minimize cognitive overload faced by users, to alleviate the disorientation problem of users, to enhance the usability and the utility of the system by applying intelligent information adaptation (personalization) techniques for user/system interactions that take into account individual differences of users [2]. Adaptation involves two key activities: (i) a user modelling activity to develop a user model and (ii) an adaptation activity that leverages a 'rich' user-model to personalize the information content, the information presentation style and the navigation path of the system to the user [3]. One of the ways to enhance the efficiency of WAHS is to build accurate user models. It can be achieved by taking into account human factors (or individual differences) that have significant effects on 
human computer interaction and on the learning process [4, 5]. Research into individual differences suggests cognitive/learning styles (CLS) have significant effects on student learning in hypermedia systems [5-8].

Information imperfection, that is, information used in one or more respects is imprecise, uncertain, incomplete, unreliable, vague or partially true, is an inherent characteristics of WAHS [9]. Imprecision of WAHS is rooted in imprecision of its input information. Sources of input information to build a user model are either the explicit information provided by subjective judgments of users/experts or the implicit information inferred by monitoring and measurement of a user behaviour, or combination of both. This imprecision is passed on user model and then on adaptation strategy that is guided by heuristics, hypotheses, or approximate decisions.

This paper deals with uncertain and vague information in WAHS and proposes fuzzy logic approach to handle it. Fuzzy granulation method is proposed to create a fuzzy inference system (FIS) to adaptively map cognitive/learning styles of users to their information navigation/presentation preferences (NPL) through natural language expressions. The important implications of this approach are that, first, uncertain and vague information used is handled; second, a mechanism for approximate adaptation at a variety of granulation levels is provided; third, a qualitative linguistic model of adaptation is presented. The proposed approach is close to human reasoning and thereby lowers the cost of solution, and facilitates the design of human computer interaction systems with high level intelligence capability.

The paper is organized as follows. The description of cognitive and learning styles is given in Section 2. Navigation and presentation preferences of users are presented in Section 3. The adaptation process, the fuzzy granulation of input and output linguistic variables, and an inference mechanism are presented in Section 4. Section 5 is devoted to related works. Finally, in Section 6 we present results and conclusion.

\section{Cognitive/Learning Styles}

Cognitive styles (CS) deal with the form of cognitive activity (thinking, perceiving, remembering), not its content. Learning styles (LS), on the other hand, is seen as a broader construct, which includes cognitive along with affective and physiological styles. A key factor in determining cognitive styles with respect to learning is the field dependency factor. Filled dependence refers to an individual's ability to perceive a local field as discrete form of its surrounding field. It is a single bi-polar dimension ranging from Field dependent (FD) individuals at one extreme to Field independent (FI) individuals at the other [10]. Some cognitive abilities are highly coupled to perceptual abilities because they are sensitive to acquisition modalities (view, hearing, touch, taste, smell) [11]. Characteristics of users in respect to $\mathrm{CL}$ are described in [7] and can be modelled by a hierarchy type tree structure given in Fig. 1.

The dimension visual/verbal of the model developed by Felder- Silverman is used to reflect the learning modalities. However, CLS is a disputable concept that is not fully accepted by the whole community. 


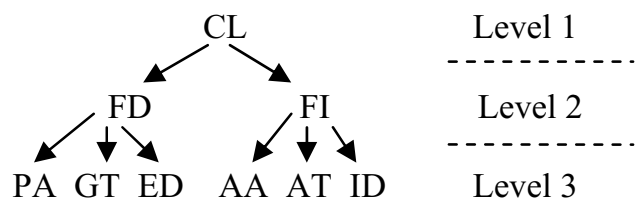

Fig. 1. Hierarchical type tree structure of CLS

where FD - field dependent, PA - passive approach, GT - global tendency, ED externally directed, FI - field independent, AA - active approach, AT - analytical tendency, ID - internally directed.

In most of the systems CLS is assessed through psychological questionnaires and psychometric tests or in the form of self-report. This kind of measures of CLS is based on subjective judgment users make about themselves. Furthermore, not all characteristics they measure are stable and invariable across different subject domains. It is often the case when the mixed result for the same person is obtained, that is a user may have preference for one particular style, preference for more than one style and different levels of preferences for the different styles $[12,13]$. For example, a learner may be attributed to the visual style at the high level, but also to verbal style up to a certain extent, at the medium level. For that reason, CLS characteristics of users are intrinsically imprecise and consist of overlapping classes of styles one can not to draw a line between them. Fuzzy logic and granulation methods are proposed in Section 4 to handle the fuzziness of CLS.

\section{Navigation and Presentation Preferences}

Navigation preferences of users in respect to cognitive styles characteristics described in Fig. 1 can be presented in the form [7]:

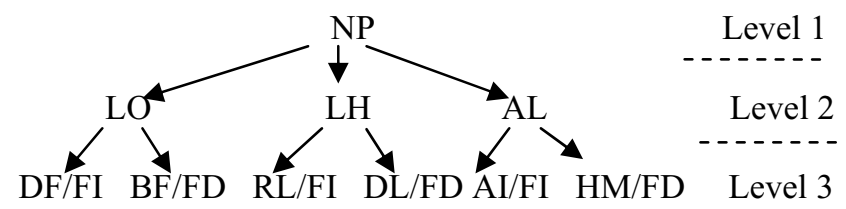

Fig. 2. Navigation preferences

where NP - navigation preference, LO - link ordering, LH - link hiding, AL adaptive layout, DF - depth firth path, FI - field independent, BF - breadth-firth path, FD - field dependent, RL - rich links, DL - disabled links, AI - alphabetical index, $\mathrm{HM}$ - hierarchical map.

Presentation preferences are modes of delivering the content using a variety of multimedia techniques such as text, graphics, image, audio, video and etc. 


\section{Adaptation to Cognitive/Learning Styles}

Two types of adaptation are distinguished in WAHS, namely adaptive presentation support and adaptive navigation support. The aim of adaptive navigation is to support users to find their learning paths in hyperspace by editing links. For example, a link can be added, removed, or edited to change its format and presentation. The adaptive presentation supports users in selecting the content of the current node of course structure and the content presentation style or mode [3]. In this study, the basic source of the adaptation information is CLS that is to be adapted to navigation and presentation preferences of users. We interpret the process of adaptation as an inference mechanism that maps a set of input variables to a set of output variables. FIS provides the formalism that maps one family of fuzzy sets to another family of fuzzy sets. This formalism serves as a means of precision of imprecise information through graduated (or fuzzy) granulation $[14,15]$.

\subsection{Fuzzy Multi Level Granulation of Input Linguistic Variables}

Let us introduce linguistic variables $C S, F D, P A, G T, E D, F I, A A, A T, I D$, associated with the concepts CS, FD, PA, GT, ED, FI, AA, AT, ID, respectively, which are described in Fig. 1. Membership functions of fuzzy sets of these linguistic variables are defined by applying multi level granulation technique. The first level granulation applied to the root $\boldsymbol{C S}$ of the tree yields:

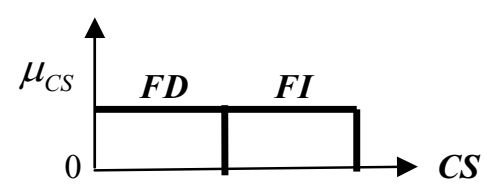

Fig. 3. Crisp sets for linguistic variable $\boldsymbol{C S}$

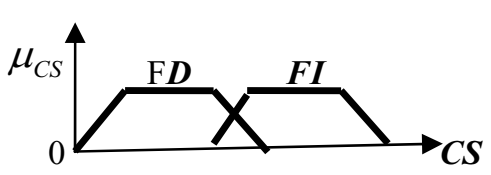

Fig. 4. Granulation of $C S$

The second level of granulation is applied to the nodes of the tree that are in the second level, for example, for $\boldsymbol{F D}$ we have:

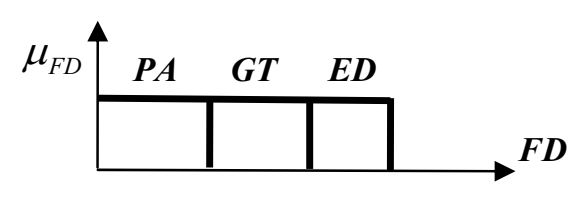

Fig. 5. Crisp sets for $\boldsymbol{F D}$

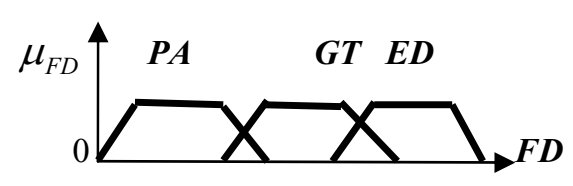

Fig. 6. Granulation of $\boldsymbol{F D}$

Finally, the granulation of the nodes from the third level through linguistic qualifiers poor, good, and excellent is shown in Fig. 7.

Next, we can use hedges for the next granularity level. Hedges are linguistic modifiers operated on membership functions. They are expressed by adjectives and/or adverbs such as very, somewhat, slightly, more or less, quite, extremely, fairly, below 
and etc. For example, hedge very applied to qualifier poor modifies its membership function as follows: $\mu_{\text {verypoor }}=\left(\mu_{\text {poor }}\right)^{2}$.

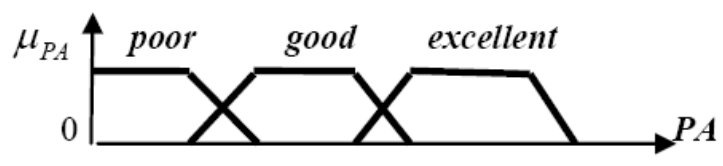

Fig. 7. Granulation of linguistic variable $\boldsymbol{P A}$

The visual/verbal styles modelled by linguistic variables $\boldsymbol{V I}$ and $\boldsymbol{V B}$, respectively, can also be granulated in the similar way.

\subsection{Fuzzy Multi Level Granulation of Output Linguistic Variables}

Based on Fig. 2 we introduce output linguistic variables $N P, L O, L H, A L, D F, B F$, $\boldsymbol{R} \boldsymbol{L}, \boldsymbol{D L}, \boldsymbol{A I}$, and $\boldsymbol{H} \boldsymbol{M}$ associated with the concepts NP, LO, LH, AL, DF, BF, RL, DL, AI, and HM, respectively. Applying multi level granulation method, similar to the one described above, we can form output membership functions for output linguistic variables $\boldsymbol{L O}, \boldsymbol{L H}$, and $\boldsymbol{A L}$ in the form shown in Fig. 8, where for the linguistic variable $\boldsymbol{L O}-\boldsymbol{A}$ stands for $\boldsymbol{D F}, \boldsymbol{B}$ stands for $\boldsymbol{B F}$; for the linguistic variable $\boldsymbol{L H}-\boldsymbol{A}$ stands for $\boldsymbol{R} \boldsymbol{L}, \boldsymbol{B}$ stands for $\boldsymbol{D} \boldsymbol{L}$; and finally, for the linguistic variable $\boldsymbol{A} \boldsymbol{L}-\boldsymbol{A}$ stands for $\boldsymbol{A I}$, B stands for $\boldsymbol{H} \boldsymbol{M}$.

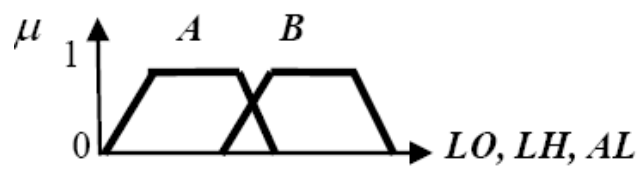

Fig. 8. Granulation of linguistic variables $\mathbf{L O}, \boldsymbol{L H}$, and $\boldsymbol{A L}$

Finally, we create one more output linguistic variable $\boldsymbol{M M M}$ associated with the concept presentation preferences and relate it with the input linguistic variables $\boldsymbol{V I} / \boldsymbol{V B}$. The multimedia mode of presentation includes text, image, audio, video, graphics, games, animation and any combination of these elements. A granulation of linguistic variable $\boldsymbol{M M M}$ can be shown as follows:

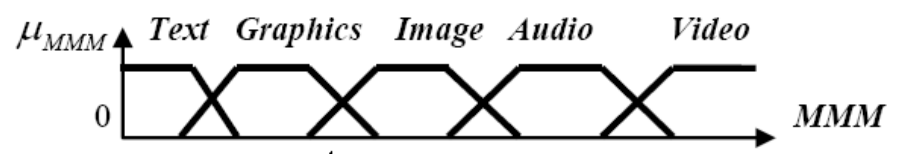

Fig. 9. Granulation of the linguistic variable MMM

Again, we can use linguistic qualifiers and modifiers, as we did with input linguistic variables, for the next granulation levels. The linguistic qualifiers low, medium, high used for output linguistic variables are shown in Table 1. 


\subsection{Inference}

Fuzzy inference is a method that interprets the values in the input vector and, based on some set of if -then rules, assigns values to the output vector. Fuzzy if - then rules capture the expert knowledge in the form of fuzzy predicates that establishes relations between input and output linguistic variables. The fuzzy predicates are associated with linguistic terms, and the proposed model is, in fact, a qualitative description of the system using rules like: IF input linguistic variable $\boldsymbol{C} \boldsymbol{L S}$ is poor THEN output linguistic variable $\boldsymbol{N P L}$ is high. Such models are often called linguistic models $[16,17]$.

Table 1. FIS for adaptation cognitive/learning styles to navigation/presentation preferences with a variety of granulation levels

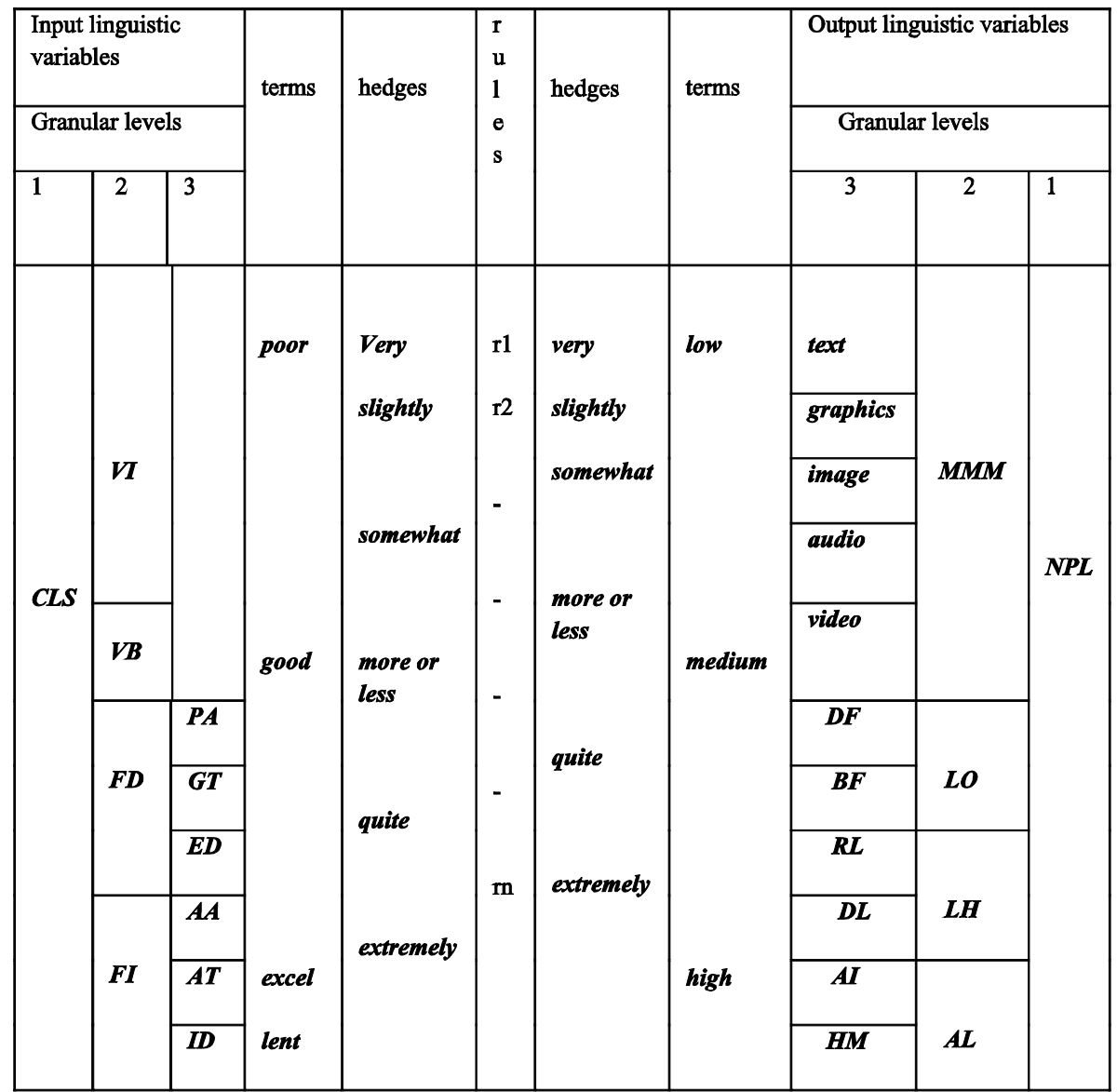

More formally, let $\boldsymbol{C L S}$ and $\boldsymbol{N P L}$ are linguistic variables defined by fuzzy sets on the universes of discourse that contain granular values described in 4.1 and 4.2. Denote membership functions of linguistic variables $\boldsymbol{C L S}$ and $N P \boldsymbol{L}$ by $\mu_{C L S}$ and $\mu_{N P L}$, 
respectively. Then the adaptation process can be characterized by a mapping $f: \mu_{C L S} \rightarrow \mu_{N P M}$. Granulation of function $f$ is a fuzzy graph that is described as a collection of if-then rules. A fuzzy if-then rule can be defined as a binary fuzzy relation $R$ considered as a fuzzy set with membership function: $\mu_{R}=f\left(\mu_{C L S}, \mu_{N P L}\right)$. Using the compositional rule of inference, we can formulate the inference procedure in fuzzy reasoning in the form: $\boldsymbol{N P \boldsymbol { L }}=\boldsymbol{C L S} \circ R$, where the sign $\circ$ denotes a fuzzy composition operator, consisting of a t-norm operator, followed by a t-conorm operator. The FIS for the adaptation of CLS to NPL is shown in Table 1. Some examples of rules at a variety of granulation level are presented below:

Level 1: IF $C L S$ is $F D$, THEN $N P L$ is $D L ;$ IF $C L S$ is $A A$, THEN $N P L$ is $D F$; IF $C L S$ is poor, THEN NPL is high; IF CLS is very poor, THEN NPL is extremely high.

Level 2: IF $F D$ is good, THEN $D L$ is medium; IF $F I$ is quite good, THEN $A I$ is somewhat medium; IF VI is excellent, THEN MMM is video; IF VI is good and VB is good, THEN MMM is video and audio.

Level 3: IF $P A$ is good and $A A$ is good, THEN $D F$ is medium and $B F$ is medium; IF $A A$ is very good, THEN $A I$ is quite high.

The number of rules increases exponentially with the number of inputs, but some rules may rarely or never occur. So, fewer rules may be predefined by experts in the domain. Moreover, one can switch between granules at different levels. It can be done using a meta rule. For example, if $X$ is a set of granules $F D, F I, V I$ and $V B$, then the following two step rule can be written: IF CLS is $X$, THEN select the rule associated with the value of $X$. Here the first rule serves as a meta rule and is used to control the order of firing rules from Level 2.

\section{Related Work}

Fuzzy modelling has previously applied with success to a variety of problems of WAHS. FIS based on a rule-based representation of user profiles is given in [18] for web recommendation system. In the educational AHS, the work [19] employs fuzzy logic for modelling user knowledge of domain. The work [20] proposed a model named MAZE to capture imprecise behaviours of users. The neuro-fuzzy approach successfully applied to the student diagnosis in [21]. The work [22] proposes an algorithm of adaptation to psychological factors using multi utility attribute theory and fuzzy set theoretic similarity measure. A survey of application of soft computing methods to modelling user behaviour is presented in [23]. The neuro-fuzzy based web recommendation system is examined in [24].

\section{Results and Conclusion}

The proposed linguistic model can be simulated using Fuzzy Logic Toolbox in Matlab. The aim is to observe the behaviour of the proposed model and tune its 
parameters such as: input and output linguistic terms and their membership function shapes; relevance and weights of rules; the type of inference mechanism - Mamdani type max-min composition or Takagi-Sugeno type linear bounded-sum; the type of defuzzification (centroid, middle of maximum, largest of maximum, and smallest of minimum). The Mamdani type FIS expects the membership functions of output linguistic variables to be fuzzy sets and requires the defuzzification step, while Takagi-Sugeno type FIS expects output membership functions to be singletons. Until now, preliminary simulation results have shown that FIS based on Mamdani max-min is preferred at a lower level of granulation, whiles Sugeno-type FIS is more appropriate at a higher level of granulation. The simulation results are in line with expectations of domain experts. As a future work, a prototype of the model shall be developed to validate the proposed linguistic model within the efficiency of user/system interactions in terms of the usability and the utility of the system. This paper has proposed a fuzzy linguistic model for adaptation to CLS based on knowledge acquisition through multi level granulation method. The implication of this approach for designing adaptive user interfaces is introducing more natural language expressions. The qualitative description of the adaptation process is close to human reasoning and thus facilitates the design of the adaptive interface, increases the interpretability, usability of the system and utility of information, saves memory space and cost expenses by computation with words instead of numbers.

\section{References}

1. Brusilovsky, P.: Adaptive Hypermedia. User Modelling and User- Adapted Interaction 11(1/2), 87-110 (2001)

2. Mobasher, B. Anand S. S.: Intelligent techniques for Web personalization, http://www.inf.unibz.it/ ricci/ATIS/papers/itwp-v5.pdf

3. Brusilovsky, P.: Methods and techniques of adaptive hypermedia. User Modelling and User- Adapted Interaction 6(2), 87-129 (1996)

4. Tsianos, N., Germanakos, P., Lekkas, Z., Mourlas, C., Samaras, G.: An assessment of human factors in adaptive hypermedia environments, pp. 1-34. IGI Global (2009)

5. Triantafillou, E., Pomportsis, A., Demetriadis, S.: The Design and the Formative Evaluation of an adaptive Educational System based on Cognitive Styles. Computers \& Education 41, 87-103 (2003)

6. Chen, S.: A cognitive model for non-linear learning in hypermedia programmes. British Journal of Educational Technology 33(4) (2002)

7. Mitchell, T., Chen, S.Y., Macredie, R.: Cognitive Styles and Adaptive Web-based Learning, http: / / bura.brunel.ac.uk/handle/2438/388

8. Tsianos, N., Germanakos, P., Lekkas, Z., Mourlas, C.: Individual differences in Adaptive educational hypermedia: the effect of cognitive style and visual working memory, pp. 147-163. IGI Global (2009)

9. Garcia, E., Sicilia, M.A.: Information imperfection as inherent characteristics of adaptive hypermedia: imprecise models of users and interactions. In: Chen, S., Magoulas, G. (eds.) Adaptable and Adaptive Hypermedia Systems, pp. 150-167. IBM Press (2010)

10. Witkin, H.A., Moore, C.A., Goodenough, D.R., Cox, P.W.: Field-dependent and Field independent Cognitive Styles and Their Educational Implications. Review of Educational Research 47, 164 (1977) 
11. Tarin-Bernard, F., Habieb-Mammar, H.: Modelling Elementary Cognitive Abilities for Adaptive Hypermedia Presentation. User Modelling and User-Adapted Interaction 15, 459-495 (2005)

12. Coffield, F., Moseley, D., Hall, E. and Ecclestone, K.: Learning Styles and Pedagogy in Post-16 Learning: a Systematic and Critical Review. Learning and Skills research centre, http: //www. Isda.org.uk/files/pdf/1543.pdf

13. Stash, N.: Incorporating Cognitive/Learning Styles in a General-Purpose Adaptive Hypermedia System. Eindhoven: Technische Universiteit Eindhoven, Proefschrift (2007), http: / /alexandria.tue.nl/extra2/200710975.pdf

14. Zadeh, L.A.: Is there a need for fuzzy logic? Information Sciences 178, 2751-2779 (2008)

15. Zadeh, L.: The concept of a linguistic variable and its applications to approximate reasoning. Part 1, Information sciences 8, 199-249 (1975), Part 2, Information Sciences, 8 301-357(1975), Part 3, Information Sciences, 9, pp. 43-80 (1975)

16. Zimmerman: Fuzzy set theory and its applications. Kluwer Academic Publishers, Dordrecht (2001)

17. Siler, W., Buckley, J.J.: Fuzzy expert systems and fuzzy reasoning. John Wiley \& Sons, Chichester (2005)

18. Nasraoui, O., Petenes, C.: Combining web usage mining and fuzzy inference for web personalization. In: Proceedings of WEBKDD 2003: Web Mining as Premise to Effective Web Applications, pp. 37-46 (2003)

19. Kavcic, A.: Fuzzy User Modelling for Adaptation in Educational Hypermedia. IEEE Transaction on Systems, Man, and Cybernetics - part C: Applications and Reviews 34(4) (2004)

20. Garcia, E., Sicilia, M.A.: Information imperfection as inherent characteristics of adaptive hypermedia: imprecise models of users and interactions. In: Chen, S., Magoulas, G. (eds.) Adaptable and Adaptive Hypermedia Systems, pp. 150-167. IBM Press (2010)

21. Stathacopoulou, R., Grigoriadou, M., Samarakou, M., Mitropoulos, D.: Monitoring student's action and using teacher's expertise in implementing and evaluating the neural networked-based fuzzy diagnostic model. Expert Systems with Applications 32, 955-975 (2007)

22. Huseyinov, I.N., Cellatoglu, A.: Adaptation based on psychological factors using fuzzy logic. In: Proceedings of Int'l Conf. e-Learning, e-Bus., EIS, and e-Gov., EEE 2010, pp. 297-303 (2010)

23. Frias-Martinez, E., Magoulas, G., Chen, S., Macredie, R.: Modelling human behaviour in user adaptive systems: Recent advances using soft computing techniques. Expert Systems with Application 29, 320-329 (2005)

24. Castellano, G., Fanelli, A., Torsello, M.: Dynamic link suggestion by a neuro-fuzzy web recommendation system. In: IADIS International Conference WWW/Internet, pp. 219-225 (2006) 